\title{
Screening for Syphilis, HIV, and hemoglobin during pregnancy in Moshi municipality, Tanzania: How is the health system performing (Short Communication)
}

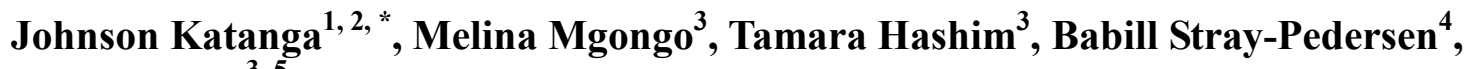 \\ Sia E. Msuya ${ }^{3,5}$ \\ ${ }^{1}$ Mirembe National hospital, Dodoma, Tanzania \\ ${ }^{2}$ Kilimanjaro Clinical Research Institute, Kilimanjaro Christian Medical university College, Moshi, Tanzania \\ ${ }^{3}$ Better Health for African Mothers and Children (BHAMC) Tanzania, Moshi, Tanzania \\ ${ }^{4}$ Div Women and Children, Rikshospitalet, Oslo University Hospital, Institute of Clinical Medicine, University in Oslo, Norway \\ ${ }^{5}$ Department of Community Medicine, Institute of Public Health, Kilimanjaro Christian Medical University College, Moshi, Tanzania
}

\section{Email address:}

johnsonkatanga2013@gmail.com (J. Katanga), linnabenny@yahoo.com (M. Mgongo), tammyhtz@yahoo.com (T. Hashim), babill.stray-pedersen@medisin.uio.no (B. Stray-Pedersen), siamsuya@hotmail.com(S. E. Msuya)

\section{To cite this article:}

Johnson Katanga, Melina Mgongo, Tamara Hashim, Babill Stray-Pedersen, Sia E. Msuya. Screening for Syphilis, HIV, and Hemoglobin during Pregnancy in Moshi Municipality, Tanzania: How is the Health System Performing (Short Communication). Science Journal of Public Health. Vol. 3, No. 1, 2015, pp. 93-96. doi: 10.11648/j.sjph.20150301.26

\begin{abstract}
The aim of the study was to determine the proportion of pregnant women that was tested for HIV, syphilis and hemoglobin during routine antenatal care in Moshi municipality, Tanzania. A cross sectional study was conducted in October 2013 - March 2014. Pregnant women in their $3^{\text {rd }}$ trimester who were attending for routine antenatal care at Pasua and Majengo health centers were enrolled. Interviews were done to determine if women were tested for the 3 tests mentioned in earlier pregnancy, prior to the study followed by clinical examination and sample collection to test for HIV, syphilis and Hb. Data were entered and analyzed by using SPSS. A total of 536 women were enrolled. Despite being in the third trimester and had attended for routine antenatal care several times, the majority of pregnant women were not screened for syphilis (89.4\%), $(28.6 \%)$ were not screened for hemoglobin level and only $1 \%$ reported not to be screened for HIV. Three hundred and sixty three participants $(87.9 \%)$ reported to have received iron supplement.
\end{abstract}

Keywords: Screening Program, HIV, Syphilis, Haemoglobin, ANC, Tanzania

\section{Introduction}

Maternal and neonatal morbidity and mortality are still a public health concern in most sub-Saharan Africa (SSA) countries including Tanzania [1]. Maternal and neonatal deaths have declined globally from 1990 to 2013; however the progress in SSA is suboptimal, as many countries have made insufficiency progress to attain the MDG 4 and $5[1,2$, 3]. Tanzania's maternal and neonatal mortality is at 454 per 100,000 live births and 26 per 1,000 live births while the target by 2015 is to reach 193 and 19 maternal and neonatal deaths respectively $[2,4,5]$. Tanzania is thus among top twenty countries with poor indicators pertaining to maternal mortality rate (MMR) and neonatal mortality rate (NMR).

Several strategies have shown positive impacts in reducing
MMR and NMR and improving the outcomes of mother and newborn babies. Among them is universal coverage of; quality antenatal care, use of SBA during pregnancy and delivery and access to emergency obstetric care (EmOC) [1, 6]. While globally the ANC coverage is $71 \%$, in Tanzania at least $98 \%$ of the women attend for at least one ANC visit, $43 \%$ attend recommended four or more ANC visits and 51\% use skilled birth attendant during birth [4]. Despite high ANC attendance, MMR and NMR are still high suggesting that quality of care offered during the antenatal care visits is suboptimal to avert morbidity \& mortality [4, 7]. TDHS of 2010 showed for example only $53 \%$ of pregnant women are counseled on danger signs, $76.2 \%$ have blood checked and $67.5 \%$ have blood pressure checked [4].

In 2002, Tanzania adopted the Focused Antenatal Care model (FANC),[8]. The FANC requires a woman without 
complications to attend for four visits with specific content related to screening, detection of complications, therapeutic interventions, and education at each visit $[8,9]$. Screening for syphilis, HIV and checking for anemia by measuring hemoglobin are among routine recommended tests to offer at first visit for every pregnant woman [8]. Syphilis is prevalent in pregnant SSA women with prevalence ranging from $4 \%$ $15 \%$ compared to prevalence of $<1 \%$ in European settings $[10,11]$. Untreated syphilis is associated with $21-50 \%$ of stillbirths in SSA, low birth weight, and congenital infections $[12,13]$. Similarly HIV in pregnancy leads to negative pregnancy outcomes like preterm delivery, low birth weight (LBW) or neonatal infections [14]. Maternal anemia is associated with increased risk of preterm delivery, LBW, maternal deaths, and neonatal anaemia [15]. Thus early screening (preferably at $1^{\text {st }}$ visit) to detect and manage syphilis, HIV and hemoglobin cannot be overemphasized in order to improve maternal and fetal outcomes.

In Tanzania few studies have evaluated coverage of the three tests among pregnant women. Recent NACP report showed the site coverage for PMTCT is $93 \%$ with $100 \%$ HIV testing coverage [16]. In 2009, the NACP had a report that only $37 \%$ were tested for syphilis [17]. There is lack of current information if the situation has improved or not. This study aimed to provide information on the proportion of pregnant women who were attending for routine ANC at Majengo and Pasua health centers that were tested for HIV, syphilis and $\mathrm{Hb}$.

\section{Materials and Methods}

This was a cross sectional study of pregnant women attending routine antenatal (ANC) at two large primary health care, Majengo and Pasua in Moshi, Tanzania. Moshi municipal is a district in Kilimanjaro region which is situated in northern part of Tanzania.

During the study every pregnant woman in their 3rd trimester who gave informed consent was invited to participate. Face to face interview was done by using a questionnaire to collect socio-demographic, socio-economic, sexual and behavioral characteristics and frequency of ANC use and the services which were offered. Interviews were followed by clinical examination (general and gynecological) then blood was taken for laboratory diagnosis of specific tests.

Venous blood sample (5mls) was used in the test of HIV and syphilis. HIV was confirmed by positive results from both Determine (Alere medical Co. 1td,357Matsuhidai Matsudo-shi China, Japan) and Uni gold test (Trinity Biotech plc,IDA business Park, Bray, Co. Wicklow Ireland), (recommended Tanzania national regime) Syphilis determine (wellkang ltd suite B, 29 Harley Street LONDON, WIG 9qR, U.K) used to test syphilis then active infection was confirmed by Rapid plasma Regain test (Span Diagnostic 1td,173-B New industrial estate, Road No 6-G, Udhna, Surat-394 210,India.) . $\mathrm{Hb}$ was measured by using HemoCue hemoglobinometer (Altenhofstrasse 80, 663886 St.
ingbert/Hassel, Germany).

Those infected with syphilis were given benzyl benzathine penicillin 2.4MU im weekly for 3 weeks and advised to bring their partner for screening and treatment, anaemia were given ferrous sulphate and folic acid some of them were referred to Kilimanjaro Christian Medical Center or St Joseph hospital and HIV-positive women were referred to care treatment clinic for possible initiation of triple therapy (tenofovil, lamivudine and efavirenz).

Data were enter and cleaned by using frequency in SPSS. Proportional was used to summarize categorical variable. Anaemia was classified on the bases of WHO 2011[18] criteria $>11 \mathrm{~g} / \mathrm{dl}$ normal, $10.9-10 \mathrm{~g} / \mathrm{dl}$ mild, $9.9-7 \mathrm{~g} / \mathrm{dl}$ moderate and $<7 \mathrm{~g} / \mathrm{dl}$ severe.

Approval for the study obtained from Kilimanjaro Christian Medical University College ethical committee (ETHICAL CLEARENCE CERTIFICATE No 605) and Moshi municipal medical committee. Pregnant women with less than 18years gave assent and their parents/guardian gave consent. Infected participants and those with anemia, with their partners got free treatment according to Tanzania treatment guideline for STIs/RTIs.

\section{Results}

Table 1. Socio-demographic characteristics and ANC visit of the participants $(n=536)$.

\begin{tabular}{ll}
\hline Variable & N (\%) \\
\hline Age (years) & $260(48.5)$ \\
$14-24$ & $230(42.9)$ \\
$25-34$ & $46(8.6)$ \\
$35-49$ & $25+5.7$ \\
Mean + SD & \\
Educational level & $13(2.4)$ \\
None & $325(60.6)$ \\
Primary & $198(37.0)$ \\
Secondary and above & \\
Marital status & $479(89.4)$ \\
Married/Cohabiting & $53(9.9)$ \\
Single & $4(0.7)$ \\
Divorced & \\
Gravida & $189(35.3)$ \\
1 & $170(31.7)$ \\
2 & $177(33.0)$ \\
$3+$ & $2(1-6)$ \\
Median (range) & \\
Number of ANC visits & $17(3.2)$ \\
1 & $143(27.3)$ \\
2 & $364(69.5)$ \\
\hline+
\end{tabular}

In this study 781 pregnant women were eligible, 245 (31.4\%) did not participate due to variable reasons like being sick, hungry, lack of permission from their partners and fear of speculum examination. 536 pregnant women participated in the study, their age ranged from 14 to 45 years with mean $( \pm \mathrm{SD})$ of $25.8( \pm 5.7)$ years. Most of them were either married or cohabiting $(89.4 \%)$, had primary education $(60.6 \%)$ and had made 3 or above ANC visit (69.5\%). Table 1 shows socio-demographic characteristics and ANC visit of the participants. 
Majority of them reported they were tested for HIV and hemoglobin, $99.0 \%$ and $71.4 \%$ respectively. Only $10.6 \%$ were tested for syphilis. Table 2 shows proportion of pregnant women who were tested for HIV, syphilis and haemoglobin during routine ANC attendance.

Forty percent $(40.5 \%)$ of the participants found to have anaemia and those with HIV infection were $6 \%$. The prevalence of mild, moderate and severe anaemia was $22.5 \%$, $15.7 \%$ and $2.3 \%$ respectively. Table 3 shows prevalence of HIV, syphilis anaemia among participants.

Table 2. Proportion of pregnant women who were tested for HIV, Syphilis and Haemoglobin during $A N C(n=536)$.

\begin{tabular}{ll}
\hline Variable & $\mathbf{N}(\mathbf{\%})$ \\
\hline HIV & \\
Yes & $528(99.0)$ \\
No & $5(1.0)$ \\
Syphilis & \\
Yes & $57(10.6)$ \\
No & $479(89.4)$ \\
Haemoglobin & \\
Yes & $260(71.4)$ \\
No & $104(28.6)$ \\
\hline
\end{tabular}

Table 3. Prevalence of HIV, Syphilis, anaemia among pregnant women $(n=535)$.

\begin{tabular}{ll}
\hline Variable & $\mathbf{N}(\%)$ \\
\hline HIV & $32(6.0)$ \\
Yes & $503(94.0)$ \\
No & \\
Syphilis & $4(0.7)$ \\
Yes & $531(99.3)$ \\
No & \\
Anaemia $(\mathrm{Hb}>11 \mathrm{~g} / \mathrm{dl})$ & $315(59.5)$ \\
Normal & $119(22.5)$ \\
Mild & $83(15.7)$ \\
Moderate & $12(2.3)$ \\
Severe & \\
Given hematenics & $319(87.9)$ \\
Yes & $44(12.1)$ \\
No & \\
\hline
\end{tabular}

\section{Discussion}

There has been a good coverage of ANC services throughout the world, however the quality of care that is provided in most of developing countries is of concern. In our study we observed most of the ANC attendees were tested for HIV and haemoglobin estimation, few of them were tested for syphilis. The findings of high response in HIV testing was also found in Kilombelo [19], this could be due to great attention paid to HIV by international donors through special programs like PMTCT and EMTCT in which all pregnant women must check up for HIV status and start medication as soon as they are found to be positive [14].

It was of a concern to note only $11 \%$ of pregnant women reported to have been tested for syphilis. This testing prevalence is much lower than that of $37 \%$ reported by the NACP in 2009, and of 33\% and 38\% reported in Zimbabwe and among SSA women $[17,20]$. Of great importance, syphilis is among the infectious disease which has adverse pregnant outcome for example abortion, congenital infection and still birth [3, 12]. And treatment with benzathine penicillin is cheap, affordable by most governments and available in most SSA health facilities [10, 20]. Low proportion of syphilis testing may be due to two key reasons. First it maybe that health providers are not aware of negative consequence of syphilis in pregnancy and do not give it similar priority to HIV [21]. Logistics of refrigeration for reagents for RPR has been mentioned as a problem of syphilis screening elsewhere [21]. Ministry of health and social welfare (MOHSW) had introduced rapid testing kits for syphilis in 2010 to avoid the problem of refrigeration. Thus frequent stock out of these testing kits has been reported by several regions. This is missed opportunity to pregnant women who were supposed to get such an important service as recommended by WHO and National Package of Essential Reproductive and Child health Intervention (NPERCHI) [8, 22]. Furthermore it is costeffective to routinely screen and treat syphilis on every pregnant woman as it has advantages of averting negative consequences on maternal health and pregnancy outcome [23, 24]. So responsible authorities should develop a system to assure that the adequate stocks of syphilis screening test and treatment are available and distributed to appropriate health facilities [10].

$28.6 \%$ were not tested for anemia, yet about $40.5 \%$ of the participants tested for haemoglobin estimation found to have anaemia. Nyamtema also showed that $25 \%$ of pregnant women with severe maternal morbidity were tested for hemoglobin [19], while TDHS showed only $76.2 \%$ women reported to be tested for hemoglobin [4]. Again with effects of anemia on pregnancy and neonates, this call for strengthening screening by using HemoCue which can be used up to dispensary levels. The need of having good care during ANC visit in which all pregnant women should be given supplements including hematenics which are crucial in improving maternal and child heath.

So quality of services provided in ANC should be of priority in order to fulfill the strategic plan for Tanzania in reduction of maternal, newborn and child death by 2015 . Also there should be an integrated system of monitoring and evaluation of HIV and syphilis screening and treatment in pregnancy, it will smoothen its delivery to ANC attendees.

The study show the proportion of pregnant women who were screened for HIV, syphilis and $\mathrm{Hb}$ which are essential component of in the focused antenatal care package.

However the method used in the screening of syphilis may miss some individual with early syphilis infection.

The interventions that is being taken by authorities to improve maternal health and reduce death of newborn, should go in line with the good quality of care during ANC. Apart from HIV, also syphilis screening and $\mathrm{Hb}$ estimation should be mandatory to all pregnant women attending ANC. 


\section{Acknowledgements}

The authors thank the Letten foundation for supporting the study. Much thanks to BHAMC staffs, Pasua and Majengo health centers staffs for their devotion during the study, as well as all participants for their time to participate in this study.

\section{Abbreviations}

FANC: Focused antenatal care

$\mathrm{Hb}$ : Haemoglobin

PMTCT: Prevention of mother to child transmission of HIV

EMTCT: Elimination of mother to child transmission of HIV

KCMUCo: Kilimanjaro Christian Medical University College

\section{References}

[1] WHO and UNICEF report 2014. Countdown report to 2015 maternal, newborn and child survival. www.countdown2015.com .Published in 2014, accessed September 212014.

[2] WHO and UNICEF report 2013. Accountability for maternal, newborn and child survival. www.countdown2015.com Published 2013, accessed September 21, 2014.

[3] World health Organization (2013). Sexually transmitted infections. Available at www.who.org

[4] NBS National bureau of statistics.Tanzania Demographic and health survey 2010, . Dar es Salaam Tanzania

[5] Ministry of health and social welfare. The national road map to accelerate reduction of maternal, newborn and child death $2008-2015 ; 2008$.

[6] Carroli, G., Rooney, C., \& Villar, J. How effective is antenatal care in preventing maternal mortality and serious morbidity? An overview of the evidence. Paediatric and perinatal epidemiology 2001, 15 Suppl 1, 1-42.

[7] World health organization. Antenatal care in developing countries 2003. Available at www.who.org

[8] Ministry of health and social welfare. Focused antenatal care malaria and syphilis in pregnancy 2009.

[9] Villar J, Callori G, Khan-Neelofur D, Piaggio GGP, Gülmezoglu AM. Patterns of routine antenatal care for lowrisk pregnancy. The Cochrane library www.thecochranelibrary.com . Published 200. Accessed October 1,2014

[10] Gloyd S, Chai S, Ann mercer M. Antenatal syphilis in sub Saharan Africa: missed opportunity for mortality reduction. Health policy and planning 2001; 16(1): 29 - 34.
[11] Swai RO., Somi GGR., Matee, MIN., Killewo J, Lyamuya, EF., Kwesigabo G. et al. Surveillance of HIV and syphilis infections among antenatal clinic attendees in Tanzania2003/2004. BMC Public health 2006, 6 (91).

[12] Watson-Jones D, Gumodoka B, Weiss H, Changalucha J, Todd $\mathrm{J}$, Mugeye $\mathrm{K}$ et al . Syphilis in pregnancy in Tanzania. II. The effectiveness of antenatal syphilis screening and single-dose benzathine penicillin treatment for the prevention of adverse pregnancy outcomes. J infect Dis 2002; 186: 948-957.

[13] Mullick S, Watson-Jones D, Beksinska M, Mabey D. Sexually transmitted infections in pregnancy: prevalence, impact on pregnancy outcomes and approach to treatment in developing countries. Sex Transm Infect 2005, 81: $294-302$.

[14] Ministry of health and social welfare. PMTCT guideline 2013. Available at www.moh.go.tz

[15] Lone FW, Qureshi RN, Emanuel F . Maternal anaemia and its impact on perinatal outcome. Tropical medicine and international health 2004, 9(4): 486 - 490.

[16] Ministry of health and social welfare (2014). PMTCT Tanzania. Available at www.moh.go.tz

[17] National Aids Control Program, HIV/AIDS/STI surveillance report United Republic of Tanzania, Ministry of Health and Social Welfare, 2009.

[18] World health organization. Haemoglobin concentration for the diagnosis of anaemia and assessment of severity 2011. Available at www.who.org

[19] Nyantema AS, Bartsch-de jong A, Urassa DP, Hagen JP, van Roosmalen J. The quality of antenatal care in rural Tanzania: what is behind the number of visit? BMC pregnancy and child health 2012, 12:70.

[20] Peeling RW, Holmes KK, Mabey D, Ronald A.Rapid test for sexually transimitted infections (STIs): The way forward. Sexually Transmitted Infection Journal 2006; 82.

[21] Gloyd S, Montoya P, Floriano F, Mariaana C, Chadreque M C, Pfeiffer $J$ et al. Scaling up syphilis antenatal screening in mozambique: Transforming policy to action. Sexually transmitted diseases 2007; 34(7): $31-36$.

[22] World health organization. Baseline report on global sexually transmitted infection surveillance 2012. Available at www.who.org

[23] Blandford JM, Gift TL, Vasaikar S, Mwesigwa-Kayongo D, Dlali P, Bronzan RN. Cost effectiveness of on-site antenatal screening to prevent congenital syphilis in rural Eastern Cape town province, Republic of South Africa. Sexually transmitted diseases 2007; 34(7): $61-66$.

[24] Terris-Prestholt F, Watson-Jones D, Mugeye K, Kumaranayake L, Ndeki L, Weiss $\mathrm{H}$ et al. Is antenatal syphilis screening still cost effective in sub-Saharan Africa. Sex transm infect 2003; 79: $375-381$. 